\title{
Research on Reconfigurable Control for a hovering PVTOL Aircraft
}

\author{
Xinli Xu and Chunwei Zhang* \\ School of Civil Engineering, Qingdao University of Technology, \\ Qingdao 266033, China \\ E-mail: lingyun308@163.com, zhangchunwei@qut.edu.cn \\ *Corresponding author
}

\section{Huosheng $\mathrm{Hu}$}

School of Computer Science and Electronic Engineering, University of Essex, Colchester CO4 3SQ, United Kingdom E-mail: hhu@essex.ac.uk

\begin{abstract}
This paper presents a novel reconfigurable control method for the planar vertical take-off and landing (PVTOL) aircraft when actuator faults occur. According to the position subsystem within the multivariable coupling, and the series between subsystems of position and attitude, a active disturbance rejection controller (ADRC) is used to counteract the adverse effects when actuator faults occur. The controller is cascade and ensures the input value of the controlled system can be tracked accurately. The coordinate transformation method is used for model decoupling due to the severe coupling. In addition, the Taylor differentiator is designed to improve the control precision based on the detailed research for tracking differentiator. The stability and safety of the aircraft is much improved in the event of actuator faults. Finally, the simulation results are given to show the effectiveness and performance of the developed method.
\end{abstract}

Keywords: Reconfigurable Control; PVTOL; ADRC; Coordinate Transformation; Actuator Faults; Taylor differentiator

Reference: to this paper should be made as follows: X.L. Xu, C.W. Zhang, and H.S. Hu, 'Research on Reconfigurable Control for a hovering PVTOL Aircraft', Int. J. Modelling, Identification and Control, Vol. x, No. x, 2018, pp. xxx-xxx.

Biography notes: Xinli Xu is currently a Post-doc at the Qingdao University of Technology of China. His research interests include reconfigurable flight control, fault tolerant control, adaptive control, structural vibration control, and structural health monitoring. He has taken part in or organized many international conferences, academic forum and academic communication. He is a member of IEEE and Shanghai Robotics Society.

Chunwei Zhang is a Professor in School of Civil Engineering, Qingdao University of Technology, China. His research interests include structural vibration control, structural health monitoring, disaster prevention and mitigation engineering and protection engineering. He has published over 200 papers in journals, books and conferences in these areas, and received a number of best paper awards. He is an Editor-in-Chief, chair, committee member or editorial board member of many journals and international conferences.

Huosheng $\mathrm{Hu}$ is a Professor in School of Computer Science \& Electronic Engineering at the University of Essex, United Kingdom, leading the robotics research. His research interests include robotics, human-robot interaction, embedded systems, mechatronics, learning algorithms, and pervasive computing. He has published over 450 papers in journals, books and conferences in these areas. He is a Fellow of IET, a Fellow of Institute of Measurement \& Control, and a chartered engineer. He has been a chair or committee member for many international conferences such as IEEE ICRA, IROS, ROBIO, ICMA, etc. He currently serves as an Editor-in-Chief for International Journal of Automation and Computing, Editor-in-Chief for Robotics, Editor-in-Chief for Digital Communications \& Networks. 


\section{Introduction}

Over the past decades, due to the increasing demands for reliability and survivability in modern aircraft, reconfigurable control has been widely developed and used in the flight control system (Rose et al. 2016; Wang et al. 2017). The planar vertical take-off and landing (PVTOL) aircraft is gaining more attention during the last few years. Many control strategies have been studied in order to fulfill the increasing safety demand and flight performance (Zhu et al. 2013; Xu et al. 2017; Zhu et al. 2018). As a non traditional design strategy, active disturbance rejection control (ADRC) was first proposed by Han Jingqing in 90s, and was introduced in English literature for the first time by Gao Zhiqiang (Han et al. 1999; Gao et al. 2001). ADRC can deal with the uncertainty of the general system and the complex nonlinear time-varying system, such as the coupling between the external disturbances, the unmodeled dynamics of the system and the unknown parameter perturbation. The most significant feature is that the disturbance can be estimated in real time by the extended state observer (ESO) and compensated by the feedback loop. So the output and stability of the whole system are adjusted (Zhao et al. 2015). In the study of process control, motion control applications and control theory, active disturbance rejection control is as widely used as PID control. It has a very obvious advantage for both high precision control and large scale integrated control. The information required for the controller only needs to be obtained from the input and output data of the object, without a precise mathematical model, and is widely used in nonlinear time-varying systems such a single input single output or multiple input multiple output (Sanjay et al. 2010; Zhao et al. 2016).

The rest of the paper is organized as follows. Section 2 is focused on the statement of the dynamic model of the PVTOL aircraft and the model decoupling. Section 3 describes the reconfigurable control strategy. Simulation results are presented and discussed in Section 4. Finally, a brief conclusion and future work are given in Section 5.

\section{Dynamic Models and Model Decoupling}

\subsection{Dynamic Model of PVTOL Aircraft}

The minimum number of state variable and input for hovering control of V/STOL aircraft is retained by using the PVTOL aircraft model as a benchmark model. Figure 1 shows the couple relationship between the roll moment and lateral thrust.

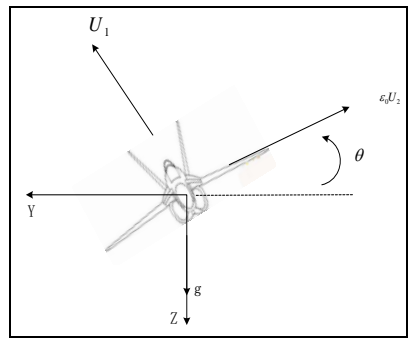

Figure1 Schematic drawing of the PVTOL aircraft

In terms of aircraft modelling, the aircraft will be considered as a rigid system and the bending effect of its fuselage and wings will be ignored. As shown in Figure 1, its motion equations can be established in the following form:

$$
\left\{\begin{array}{l}
\ddot{x}=-u_{1} \sin \theta+\varepsilon u_{2} \cos \theta+\omega_{1} \\
\ddot{y}=u_{1} \cos \theta+\varepsilon u_{2} \sin \theta-1+\omega_{2} \\
\ddot{\theta}=u_{2}
\end{array}\right.
$$

where $x$ and $y$ are the horizontal and vertical motion of aircraft mass center after simplification; $\theta$ is the roll angle which is relative to the $x$ axis; $u_{1}$ and $u_{2}$ are respectively the main thrust and roll torque control; $\varepsilon \in[0,1]$ is the coupling coefficient between the lateral thrust and roll torque and immeasurable.

Remark $1 \quad u_{1}$ and $u_{2}$ should be not too big due to the limitation of aircraft jet engines control and $u_{1}>0 \cdot y(t)$ should have a very small amount of change or $y(t)=0$.

\subsection{Model Decoupling}

Consider the control matrix with coupling characteristics of the actual PVTOL system (1), the controlled variable $\tilde{u}_{1}$ and $\tilde{u}_{2}$ are led into, as follows:

$$
\left\{\begin{array}{l}
u_{1}=-\left(\tilde{u}_{1}-\omega_{1}\right) \sin \theta+\left(\tilde{u}_{2}-\omega_{2}+1\right) \cos \theta \\
u_{2}=\frac{1}{\varepsilon}\left(\tilde{u}_{1}-\omega_{1}\right) \cos \theta+\left(\tilde{u}_{2}-\omega_{2}+1\right) \sin \theta
\end{array}\right.
$$

Then the PVTOL model (1) can be converted into:

$$
\left\{\begin{array}{l}
\ddot{x}=\tilde{u}_{1} \\
\ddot{y}=\tilde{u}_{2} \\
\ddot{\theta}=\tilde{u}_{2}
\end{array}\right.
$$

We define:

$\left[\begin{array}{c}\tilde{u}_{1} \\ \tilde{u}_{2}\end{array}\right]=\left[\begin{array}{cc}\bar{u}_{1}-\varepsilon \dot{\theta}^{2} & \varepsilon \bar{u}_{2} \\ \bar{u}_{1}-\varepsilon \dot{\theta}^{2} & \varepsilon \bar{u}_{2}\end{array}\right]\left[\begin{array}{cc}\sin \theta & -\cos \theta \\ \cos \theta & \sin \theta\end{array}\right]+\left[\begin{array}{c}\omega_{1} \\ \omega_{2}-1\end{array}\right]$ 


$$
\left[\begin{array}{l}
x \\
y
\end{array}\right]=\left[\begin{array}{l}
\tilde{x} \\
\tilde{y}
\end{array}\right]+\left[\begin{array}{l}
\varepsilon \sin \theta \\
-\varepsilon \cos \theta
\end{array}\right]
$$

Hence, the PVTOL aircraft model (3) can be transformed to:

$$
\left\{\begin{array}{l}
\ddot{\tilde{x}}=\bar{u}_{1} \sin \theta+\omega_{1} \\
\ddot{\tilde{y}}=-\bar{u}_{1} \cos \theta-1+\omega_{2} \\
\ddot{\theta}=\bar{u}_{2}
\end{array}\right.
$$

\section{Control Strategies}

In the past twenty years, ADRC has been successfully applied to many engineering fields to solve control problems, such as non circular machining, fault diagnosis, high performance motion control, chemical process control, aircraft control, robot control, and so on (Jin et al. 2013; Guo et al. 2015). The active disturbance rejection controller (ADRC) mainly includes three parts (Han et al. 1999). The first one is the tracking differentiator (TD). As a part of the control theory, it has been widely studied, and the convergence of the nonlinear tracking differentiator is also proved. As the second part of the ADRC, the extended state observer is mainly used to estimate the state of the system and the total disturbance. The convergence of the observer for the nonlinear expansion state of the single input and single output system is proved by Guo Baozhu (Huang et al. 2001; Xia et al. 2011; Guo et al. 2013). The extended state observer can also be widely used as a state observer in control theory, such as fault diagnosis. The last part is nonlinear state error feedback control law (NLSEF).

In this paper, the Cascade ADRC is designed in the channel where $x(t)$ has a strong coupling with $\theta$, to sustain the stability of the hovering aircraft during lateral maneuver when the fault occurs. Figure 2 shows the system block diagram of the Cascade ADRC method.

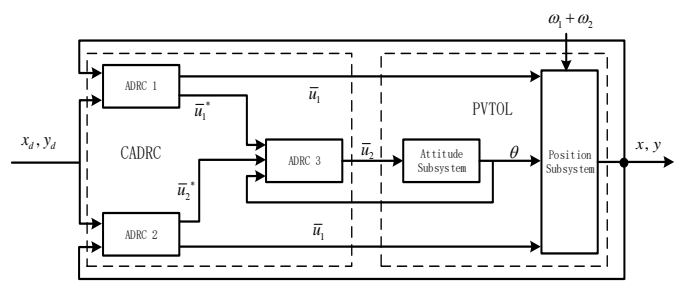

Figure 2 The schematic of the proposed FTC

In this paper, the Taylor differentiator is designed as follows:

$$
\left\{\begin{array}{l}
\dot{v}_{1}=v_{2} \\
\dot{v}_{2}=\frac{2}{\varepsilon^{2}}\left[v_{1}-v\right]-\frac{2}{\varepsilon} v_{2}
\end{array}\right.
$$

where $v$ is given signal, $v_{1}$ is tracking signal, $v_{2}$ is tracking speed, - is convergence rate.

The formula (6) can be regarded as the two parts of the position subsystem and the attitude subsystem, which constitute the outer ring and inner loop of the system respectively. The two are series connection and can be expressed as:

$$
\begin{gathered}
\left\{\begin{array}{l}
\ddot{\tilde{x}}=\bar{u}_{1} \sin \theta+\omega_{1} \\
\ddot{\tilde{y}}=-\bar{u}_{1} \cos \theta-1+\omega_{2}
\end{array}\right. \\
\left\{\begin{array}{l}
\dot{\theta}=\tilde{\theta} \\
\dot{\tilde{\theta}}=\bar{u}_{2}
\end{array}\right.
\end{gathered}
$$

Defining:

$$
\ddot{x}_{1}=\ddot{\tilde{x}}, \ddot{x}_{2}=\ddot{\tilde{y}}
$$

Then the formula (8) can be transformed to:

$$
\left\{\begin{array}{l}
\ddot{x}_{1}=\bar{u}_{1} \sin \theta+\omega_{1} \\
\ddot{x}_{2}=-\bar{u}_{1} \cos \theta-1+\omega_{2} \\
y_{1}=x_{1} \\
y_{2}=x_{2}
\end{array}\right.
$$

We have that,

$$
\begin{gathered}
B=\left[\begin{array}{c}
\sin \theta \\
-\cos \theta
\end{array}\right] \\
{\left[\begin{array}{cc}
\bar{u}_{1}^{*} & \bar{u}_{2}^{*}
\end{array}\right]^{T}=B \bar{u}_{1}}
\end{gathered}
$$

where $\bar{u}_{1}{ }^{*}$ and $\bar{u}_{2}{ }^{*}$ is the virtual controlled variable respectively.

For the position subsystem (8), which is a $2 \times 2$ coupled system, the ESO can be established respectively, as follows:

$$
\begin{aligned}
& \left\{\begin{array}{l}
e=z_{11}-x_{1} \\
\dot{z}_{11}=z_{12}-\beta_{01} e \\
\dot{z}_{12}=z_{13}-\beta_{02} f a l(e, 0.5, h)+\bar{u}_{1}^{*} \\
\dot{z}_{13}=-\beta_{03} f a l(e, 0.25, h)
\end{array}\right. \\
& \left\{\begin{array}{l}
e=z_{21}-x_{2} \\
\dot{z}_{21}=z_{22}-\beta_{01} e \\
\dot{z}_{22}=z_{23}-\beta_{02} f a l(e, 0.5, h)+\bar{u}_{2}^{*} \\
\dot{z}_{23}=-\beta_{03} f a l(e, 0.25, h)
\end{array}\right.
\end{aligned}
$$


where $\beta_{01}, \beta_{02}$ and $\beta_{03}$ are the parameters of observer which need to determine. $z_{11}, z_{12}, z_{13}$, $z_{21}, z_{22}$ and $z_{23}$ are the input variables of ESO. $f a l(e, 0.5, h)$ and $f a l(e, 0.25, h)$ are the nonlinear function. $h$ is the integral step. The system will be tracked by $z_{11}, z_{12}, z_{13}, z_{21}, z_{22}$ and $z_{23}$ when the ESO in (14) is tuned properly.

Then the virtual controlled variable $\bar{u}_{1}^{*}$ and $\bar{u}_{2}{ }^{*}$ can be obtained:

$$
\begin{aligned}
& \left\{\begin{array}{l}
e_{1}=v_{11}-z_{11} \\
e_{2}=v_{12}-z_{12} \\
\bar{u}_{1}^{*}=-\operatorname{fhan}\left(e_{1}, e_{2}, r, h_{1}\right)-z_{13}
\end{array}\right. \\
& \left\{\begin{array}{l}
e_{1}=v_{21}-z_{21} \\
e_{2}=v_{22}-z_{22} \\
\bar{u}_{2}^{*}=-\operatorname{fhan}\left(e_{1}, e_{2}, r, h_{1}\right)-z_{23}
\end{array}\right.
\end{aligned}
$$

The actual control variable can be obtained by the formula (11), (12), (15) and (16):

$$
\bar{u}_{1}=B^{-1}\left[\begin{array}{ll}
\bar{u}_{1}^{*} & \bar{u}_{2}^{*}
\end{array}\right]^{T}
$$

From above, the attitude subsystem (9) and the position subsystem are series connected, and then the ESO can be established as follows:

$$
\left\{\begin{array}{l}
e=z_{31}-\theta \\
\dot{z}_{31}=z_{32}-\beta_{01} e \\
\dot{z}_{32}=z_{33}-\beta_{02} \operatorname{fal}(e, 0.5, h)+\bar{u}_{2} \\
\dot{z}_{33}=-\beta_{03} \operatorname{fal}(e, 0.25, h)
\end{array}\right.
$$

After disturbance compensation, the control variable can be obtained by the error feedback control law:

$$
\left\{\begin{array}{l}
e_{1}=U-z_{31} \\
e_{2}=-z_{32} \\
\bar{u}_{2}=\operatorname{fhan}\left(e_{1}, e_{2}, r, h_{2}\right)-z_{33}
\end{array}\right.
$$

where $U=\bar{u}_{1}^{*}+\bar{u}_{2}^{*}$.

As mentioned above, $\beta_{01}, \beta_{02}$ and $\beta_{03}$ need to be tuned. (Gao et al. 2001) uses the bandwidth theory to determine the relevant parameters. For example, the characteristic equation of ESO (16) can be expressed, as:

$$
D(s)=s^{3}+\beta_{01} s^{2}+\beta_{02} s+\beta_{03}
$$

It is shown that the estimator is better when the characteristic equation (20) is stable and then the equation coefficients meet the requirements.

Defining:

$$
D(s)=\left(s+\omega_{0}\right)^{3}
$$

Then $\beta_{01}, \beta_{02}$ and $\beta_{03}$ can be converted to the function of the observer bandwidth $\omega_{0}$ :

$$
\left\{\begin{array}{l}
\beta_{01}=3 \omega_{0} \\
\beta_{02}=3 \omega_{0}^{2} \\
\beta_{03}=\omega_{0}^{3}
\end{array}\right.
$$

Hence, we will obtain a good performance for Cascade ADRC by tuning the parameters.

\section{Control Strategies}

In this section, the MATLAB software is used for a couple of numerical simulations. For comparison, the results under normal and fault are presented simultaneously. The initial condition of the aircraft is $\left[\begin{array}{lll}x_{0} & y_{0} & \theta_{0}\end{array}\right]^{T}=\left[\begin{array}{lll}0 & 0 & 0\end{array}\right]^{T}$. Two scenarios are considered in the numerical simulations: a jammed $5^{\circ}$ and $10^{\circ}$ in elevator respectively.

As shown in Figure 3, the aircraft has normal flight in the first 20 seconds. When the elevator is jammed at 5 and 10 degrees respectively, the response curve of the system is shown as follows:
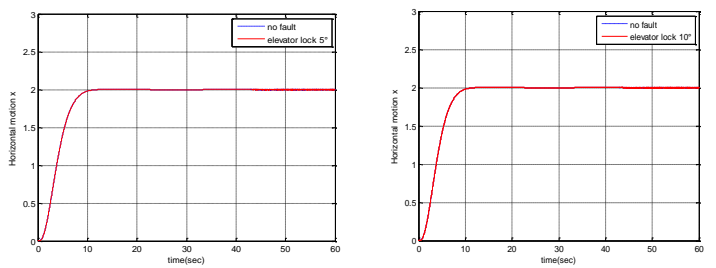

(a) $\mathrm{X}$ (the horizontal output)
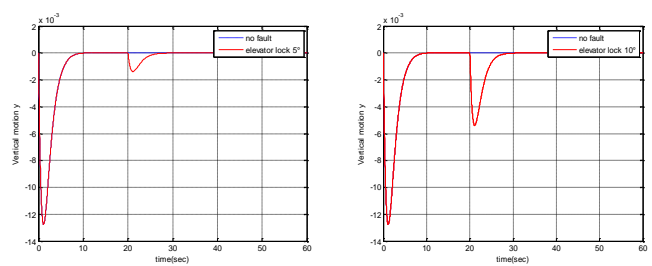

(b) $\mathrm{y}$ (the vertical output)
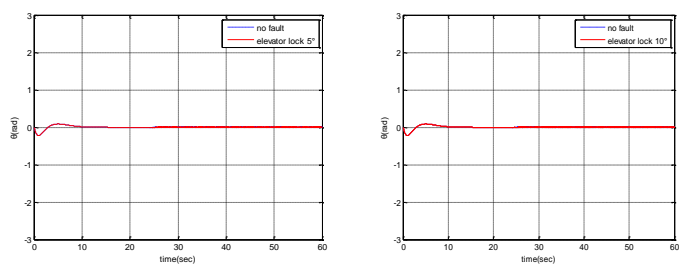

(c) $\theta$ (the roll angle output)

Figure 3 The system output when the elevator is jammed

When the elevator is jammed at 5 degrees, the horizontal displacement and roll angle of the aircraft 
are less affected, and the vertical displacement is greatly influenced. When the elevator is jammed at 10 degrees, the horizontal displacement and the roll angle oscillation are still not obvious, while the vertical displacement is larger than the former. From Figure 4, we can see that the impact of aircraft jammed fault on aircraft is relatively large. At this time, using the method proposed in this chapter, the adjustment time of the system will be shortened and the amplitude of the oscillation will be reduced. The system will be stable soon, and the aircraft will return to the expected position after a short wave of fluctuation. It shows that the reconfigurable control method is effective in fault of the actuator jammed, and the system performance is good and stable.

\section{Conclusions and Future Work}

A cascade active disturbance rejection controller is presented to accommodate partial loss fault for the PVTOL aircraft. According to the position subsystem within the multivariable coupling, and the series between subsystems of position and attitude, a active disturbance rejection controller (ADRC) is used to counteract the adverse effects when actuator faults occur. In addition, the Taylor differentiator is designed to improve the control precision based on the detailed research for tracking differentiator. Jams in an elevator are considered and the resulting control law is validated in the numerical simulations. The results demonstrate the satisfactory performance and robustness of the proposed method in the event of actuator failures.

The future work is to apply the presented method in a VTOL aircraft which is no longer a simplified version. The performance and effectiveness will be tested in an actual aircraft model and then it could be used in real aircrafts for a variety of tasks.

\section{REFERENCES}

Rose J.B.R. and Jinu G.R. (2016). 'Influence of aeroelastic flow induced oscillations on fatigue life of an airplane wing structure', International Journal of Modelling, Identification and Control, Vol. 25, No. 3, pp. 199-216.

Wang, F. , Oskoei, M. , \& Hu, H. . (2017). 'Multi-finger myoelectric signals for controlling a virtual robotic prosthetic hand', International Journal of Modelling Identification \& Control, Vol. 27, No. 3, pp.181.

Quanmin Zhu, Li Liu, Weicun Zhang, and Shaoyuan Li. (2018). 'Control of Complex Nonlinear Dynamic Rational Systems', Complexity, Vol. 2018

Zhu, P. , Xu, B. , \& Lu, M. . (2013). 'An Intelligent Fusion Algorithm for Uncertain Information Processing. Advances in Swarm Intelligence', Springer Berlin Heidelberg.

Xu, X. , Jiang, Z. , \& Hu, H. . (2017). 'A novel reconfigurable control method for an aircraft with potential actuator failures', International Journal of Computer Applications in Technology, Vol. 56, No. 3, pp.163.

J. Han. (1999). 'Nonlinear design methods for control systems', Proc. of the 14th IFAC World Congress.

Z. Gao, Y. Huang, and J. Han. (2001). 'An alternative paradigm for control system design', Proc. of IEEE conference on Decision and Control, Vol. 5, No.4, pp.4578 $-4585$

Zhao, Z. L. , \& Guo, B. Z. . (2015). 'On active disturbance rejection control for nonlinear systems using time-varying gain', European Journal of Control, 23, 62-70.

Sanjay E. Talole, Jayawant P. Kolhe, and Srivijay B. Phadke.. (2010). 'Extended-State-Observer-Based Control of Flexible-Joint System With Experimental Validation', IEEE Transactions On Industrial Electronics, Vol. 57, No. 4, pp.1411-1419.

Zhao, Z. L. , \& Guo, B. Z. . (2016). 'Active disturbance rejection control approach to stabilization of lower triangular systems with uncertainty', International Journal of Robust \& Nonlinear Control, Vol. 26, No. 11, pp.23142337.

Bao - Zhu Guo, \& Jun - Jun Liu. (2015). 'Sliding mode control and active disturbance rejection control to the stabilization of one - dimensional schrödinger equation subject to boundary control matched disturbance', International Journal of Robust \& Nonlinear Control, Vol. 24, No. 16, pp. 2194-2212.

Guo, B. Z. , \& Jin, F. F. . (2013). 'Sliding mode and active disturbance rejection control to stabilization of onedimensional anti-stable wave equations subject to disturbance in boundary input', IEEE Transactions on Automatic Control, Vol. 58, No. 5, pp.1269-1274.

Guo, B. Z. , \& Jin, F. F. . (2013). 'The active disturbance rejection and sliding mode control approach to the stabilization of the euler-bernoulli beam equation with boundary input disturbance', Automatica, Vol. 49, No. 9, pp.2911-2918.

Yi Huang, Kekang Xu, Jingqing Han and James Lam. (2001). 'Flight Contrl Design Using Extended State Observer and Nonsmooth Feedback', In the proc. of 40th IEEE Congerence On Decision and Control, pp. 223-228.

Xia, Y. , Zhu, Z. , Fu, M. , \& Wang, S. . (2011). 'Attitude tracking of rigid spacecraft with bounded disturbances', IEEE Transactions on Industrial Electronics, Vol. 58, No. 2, pp.647-659. 